STUDI

FRANCESI
Studi Francesi

Rivista quadrimestrale fondata da Franco Simone

143 (XLVIII | II) | 2004

Varia - fasc. II - maggio-agosto 2004

\title{
Lire «Les Fleurs du Mal». Textes réunis par José-Luis Diaz
}

\section{Mario Richter}

\section{(2) OpenEdition}

\section{Journals}

\section{Édition électronique}

URL : https://journals.openedition.org/studifrancesi/39691

DOI : 10.4000/studifrancesi.39691

ISSN : 2427-5856

Éditeur

Rosenberg \& Sellier

\section{Édition imprimée}

Date de publication : 1 décembre 2004

Pagination : 400-403

ISSN : 0039-2944

\section{Référence électronique}

Mario Richter, «Lire «Les Fleurs du Mal». Textes réunis par José-Luis Diaz », Studi Francesi [En ligne], 143 (XLVIII | II) | 2004, mis en ligne le 30 novembre 2015, consulté le 19 mai 2021. URL : http:// journals.openedition.org/studifrancesi/39691; DOI : https://doi.org/10.4000/studifrancesi.39691

Ce document a été généré automatiquement le 19 mai 2021.

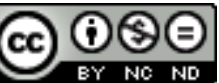

Studi Francesi è distribuita con Licenza Creative Commons Attribuzione - Non commerciale - Non opere derivate 4.0 Internazionale. 


\title{
Lire «Les Fleurs du Mal». Textes réunis par José-Luis Diaz
}

\author{
Mario Richter
}

\section{RÉFÉRENCE}

Lire «Les Fleurs du Mal». Actes des Journées d'étude organisées à Paris 7 par la Société des études romantiques, 10 au 11 octobre 2002, Textes réunis par José-LuIs DIAZ, Cahiers

Textuel nº 25, 2002. pp. 272.

1 Motivé par la présence des Fleurs du Mal au programme de l'Agrégation, José-Luis Diaz a rassemblé dans ce volume un groupe de dix-sept spécialistes capables d'approfondir un certain nombre d'aspects significatifs de l'œuvre poétique de Baudelaire, aspects qu'il a subdivisés en cinq sections thématiques.

2 La première section, consacrée aux Paradoxes et métamorphoses de l'art, comporte cinq contributions.

On commence par une étude de Patrick LABARTHE (Les onze premières «Fleurs du Mal» ou les paradoxes de l'art, pp. 11-21). Soulignant la nature réflexive de la poésie baudelairienne et passant à travers des textes fondamentaux tels que J'aime le souvenir de ces époques nues, Le mauvais moine, L'Ennemi et Le Guignon, l'auteur s'est attaché à examiner l'hypothèse selon laquelle, chez Baudelaire, «l'essence de la réalité est double, et [...] du coup, le rapport à cette réalité est foncièrement ambivalent». Cependant, en ce qui concerne le fameux «obscur Ennemi» de «L'Ennemi», LABARTHE - qui déclare s'intéresser précisément aux paradoxes baudelairiens - a préféré passer sous silence l'ambiguïté bien connue de l'expression, optant pour un seul de ses sens possibles, celui du Temps (sur toute cette question, nous nous limitons ici, pour faire bref, à renvoyer à notre propre contribution «Le mauvais moine» et l'«obscur Ennemi», dans Baudelaire, «Les Fleurs du Mal». L'intériorité de la forme, Sedes, 1989, pp. 105-118, récemment reprise dans notre commentaire Les Fleurs du Mal. Lecture intégrale, Genève, Slatkine, 2001). 
Parce que le programme de l'agrégation des lettres comprend aussi de façon explicite les pièces condamnées de la première édition des Fleurs du Mal (1857), SERGE MEITINGER (Censure et architecture. La place des pièces condamnées dans l'économie des «Fleurs du Mal», pp. 23-30) s'est proposé «de tenter une relecture rapide de la situation de ces pièces dans l'édition originale afin d'en évaluer l'importance stratégique et d'analyser, quand c'est le cas, l'effet de la censure sur l'architecture de la seconde édition». Il en est arrivé à la conclusion générale que voici: «Les exigences de la censure et l'évolution du poète (frustré mais aussi stimulé) ont modifié l'architecture du livre et son sens, vouant plus nettement la femme dont s'imposent surtout, et d'abord, la présence et la prestance physiques à la vindicte morale et aux tortures de l'oxymore, dressant le piédestal un peu compassé, trop convenu et convenable, de la femme idéale, éliminant les mystes [sic] de Sapho, alors que [...] sur tous ces points, la premières édition était plus nuancée et équilibrait sans doute mieux les forces, les sources et les valeurs 'en une ténébreuse et profonde unité"«.

DOMINIQUE COMBE (Régions de la poésie pure..., pp. 31-42) s'est interrogé sur la notion de l'expression "poésie pure» que Baudelaire employa au moins à quatre reprises avant que Mallarmé et Valéry n'en fassent une bannière esthétique à succès. Il a clairement montré l'incompatibilité, chez Baudelaire, de la «poésie pure» et de tout ce qui a trait à l'enseignement, à la science, à la philosophie et à la morale. Il n'empêche - souligne l'auteur - que Baudelaire admet et même admire certains poèmes philosophiques de Hugo (La Pente de la rêverie, par ex.), dans lesquels le poète s'est révélé capable de réconcilier poésie et philosophie, sachant échapper aussi bien au didactique qu'au descriptif au profit de l'imagination lyrique.

Prenant comme exemples, entre autres, des poèmes tels qu' «Elévation», «Don Juan aux enfers» «Le Rebelle», «Horreur sympathique» et «Le Cygne», JEAN-LouIS CABANÈS («Les Fleurs du Mal» et les métamorphoses du sublime, pp. 43-55) a bien éclairé l'esthétique baudelairienne du sublime, qui semble se situer à l'opposé de la tradition du beau idéal telle que Winckelmann l'énonça. La Recherche philosophique sur l'origine de nos idées du beau et du sublime (1757) de Burke, l'Essai sur les révolutions de Chateaubriand et la Critique de la faculté de juger de Kant constituent les points de repère fondamentaux permettant à l'auteur d'expliquer l'évolution du sublime baudelairien dans sa spécificité, un sublime joignant l'ironie, la mélancolie et la cruauté, défaisant l'alliance du beau et du bien (célébrée dans «Parfum exotique» et «J'aime le souvenir de ces époques nues»), s'avérant «indissociable [...] d'un échec, d'un manque, d'un exil, éventuellement même d'une défaite» et devenant par là une forme essentielle de la modernité. $\mathrm{Si}$, au sujet de «La servante au grand cœur...», CABANÈs semble voir «une autre forme de sublimité» liée à l'élégie, nous nous permettons de souligner que ce poème est éminemment sublime, précisément au sens ici illustré, car il nous semble allier de la façon la plus atroce la piété et le sarcasme (voir, à ce propos, notre propre interprétation dans Baudelaire, «Les Fleurs du Mal». Lecture intégrale, cité, t. II, pp. 1157 sq.).

7 Dans la dernière étude de cette première section, MICHEL SANDRAS (La prose du poème, pp. 57-68) a remis en discussion certains jugements célèbres selon lesquels Baudelaire serait le créateur d'une langue poétique pure, musicale et solidement composée (Valéry, Jakobson, Bonnefoy...) pour rétablir le fait que le poète des Fleurs «introduit dans ses vers de nombreux effets de prose, et cela sur différents plans: langue, versification, point de vue». A travers une série de vérifications ponctuelles, l'auteur 
conclut que Baudelaire crée un nouvel idiome poétique «laissant passer dans un certain nombre de ses poèmes les aspects qu'il jugeait les plus distinctifs de la prose narrative et les plus éloignés de la poésie pure». Cependant, précise l'auteur, «ce qui ressort en fin de compte de la lecture des Fleurs $d u$ Mal, c'est moins l'accablement du poète que sa révolte [...] c'est l'énergie [...] qu'il met en œuvre pour composer des vers».

La deuxième section («Le mal, les femmes et le poète») comporte trois volets.

ERIC BENOÎT (Extraire la beauté du Mal?, pp. 71-90) a accompli une traversée des Fleurs pour montrer d'abord que la beauté, chez Baudelaire, est double, à la fois spirituelle et matérielle, éternelle et mortelle, idéale et sensible. Il a illustré ensuite le processus d'extraction de la beauté du Mal, processus qui consisterait, selon l'auteur, en une transfiguration rédemptrice du Mal, ce qui n'empêcherait pas qu'il y ait également, dans les Fleurs, une fascination esthétique pour le Mal et même une absorption de la beauté dans le Mal.

10 A travers notamment une relecture du prologue «Au Lecteur» et de «Bénédiction», CHRISTINE PLANTÉ (Hypocrite lectrice, pp. 91-105) s'est ensuite interrogée sur les raisons qui ont porté Baudelaire à ne s'adresser qu'aux 'frères' du poète (les hypocrites lecteurs'), ce qui semblerait exclure ses 'sœurs' (les hypocrites lectrices'). Après avoir attentivement analysé, dans les Fleurs, la présence et le statut des femmes considérées sous leurs divers aspects et dans la complexité de leurs nombreuses nuances et contradictions, Christine Planté finit par affirmer que, s'il est vrai que Baudelaire rejette les lectrices en tant que telles, il est également vrai que «la femme, les femmes, demeurent nécessaires - dans leur exclusion même - à l'écriture poétique».

11 Pour saisir la spécificité du sujet baudelairien et de son fonctionnement, HENRI SCEPI (Le sujet des «Fleurs du Mal», pp. 107-121) s'est concentré sur l'«opposition forme/affect», sur l'effort concerté luttant contre la subjectivité, ce qui ferait du poème «le lieu d'une tension productive». Appelant de ses vœux quelques suggestions de Walter Benjamin et se penchant sur une série de poèmes tout particulièrement significatifs (le troisième «Spleen», «La Cloche fêlée», «J'aime le souvenir...», «Hymne à la Beauté», «Mœsta et errabunda», «L'Irrémédiable», «L'Horloge», etc.), le critique a conclu ses réflexions par l'observation suivante: «Ni psychologique, ni rhétorique, ni biographique, ni métaphysique, le sujet des Fleurs du Mal est d'abord une instance poétique critique, résolument historicisée, qui hante le langage, défait la forme, décompose la poésie».

La section intitulée «Photoscopies» regroupe deux nouvelles lectures de «Paysage» et du «Rêve d'un curieux».

13 La première est de STEVE MURPHY (Equivoques d'un 'paysage' baudelairien, pp. 125-146), un chercheur qui se révèle posséder, plus que tout autre, une connaissance à la fois complète et approfondie de la bibliographie critique consacrée au sujet. Soustrayant d'une part le poème liminaire des «Tableaux parisiens» aux interprétations courantes («dyptique euphorique - résume-t-il - dont le sens explicite exprimerait, de manière univoque, les intentions de l'auteur empirique Baudelaire») et essayant d'autre part de le lire dans son historicité (vu qu'il a d'abord paru dans «Le Présent» du 15 novembre 1857 pour être ensuite repris, en 1861, dans la deuxième édition del Fleurs du Mal), il s'est livré à une argumentation particulièrement dense et fine (réfractaire à toute réduction) pour affirmer que «Paysage», ayant eu une signification politique en 1857, aurait été soumis en 1861 à une profonde transformation. Devant ceux qui considèrent ce texte comme un mélange de sérieux et d'ironie (HAмоN) ou comme une expression de 
l'ambivalence baudelairienne (LABARTHE), MURPHY s'est demandé, pour conclure, «si le texte n'est pas, en partie, aussi bien une autocritique qu'une réaction sardonique à des poèmes comme celui de Gautier».

Dans la seconde contribution de cette section, JÉRôME THÉLOT (Le Rêve d'un curieux, ou la photographie comme Fleur du Mal, pp. 147-160) s'est efforcé de proposer une interprétation suggestive du "Rêve d'un curieux», essentiellement différente de la «lecture accomplie exemplairement par Jean Starobinski». Soulignant l'importance de la personnalité du destinataire (Félix Nadar, un nom dont n'apparaissent que les initiales, F.N., dans les Fleurs de 1861) et s'appuyant sur les jugements négatifs que Baudelaire exprima, au sujet de la photographie, dans le Salon de 1859, il soutient avec élégance et subtilité l'hypothèse selon laquelle «Le Rêve d'un curieux» - dont il fait une analyse assez minutieuse - rapporterait une séance de pose chez Nadar, ce qui intensifierait le conflit entre Poésie et Photographie (étant donné que l'identification $\mathrm{du}$ dédicataire de la part du lecteur est tout à fait indispensable pour que le raisonnement de тнÉLOT tienne debout, j'avoue que l'explication qu'il donne des initiales de la dédicace en tant que «logogriphe comme la photographie» me semble être - en dépit de l'intérêt indéniable qu'elle suscite - quelque peu captieuse).

Dans la section «L'ombre et le miroir», se donnent à lire également deux contributions.

Dans celle de PASCALE AURAIX-JONCHIÈRE (Baudelaire et les déités de l'ombre, pp. 163-173) sont examinés avec attention «Sed non satiata», «De profundis clamavi», «Le Vampire» et «La Béatrice» en raison du lien étroit qui semble rattacher deux à deux ces quatre poèmes, où Proserpine et Béatrice apparaissent comme des «déités de l'ombre», comme des déesses d'outre-tombe curieusement interchangeables, images infernales de l'«éternelle Vénus» permettant d'illustrer d'intéressants rapports du poète moderne à l'amour.

17 L'intervention de JAMES HIDDLESTON (Baudelaire au miroir, pp. 175-186) nous fait passer à travers plusieurs textes baudelairiens (pas uniquement les Fleurs) pour nous montrer comment, chez Baudelaire, «le sentiment de la profondeur est inséparable de l'effet de scintillement et de miroitement qu'il connote» et combien Baudelaire est «le créateur d'un univers, poésie et critique, dont la cohérence et l'unité sont si lumineuses que le lecteur qui s'y promène a l'impression de visiter un vaste palais de miroirs».

18 La dernière section (Baudelaire et $\mathrm{Cie}$ ) nous propose cinq études où il est surtout question des rapports que Baudelaire entretint avec d'autres poètes ou écrivains.

On commence par les pages que PIERRE BRUNEL (Baudelaire et l'homme d'Ovide', pp. 189-209) a consacrées à l'expression bien connue, «l'homme d'Ovide», que l'on lit dans Le Cygne. Faisant référence aux lectures célèbres de Jean Starobinski, les nombreuses et stimulantes réflexions de Brunel au sujet du Cygne (associées à celles qui portent sur Alchimie de la douleur et sur Horreur sympathique, ce dernier poème mettant également en scène Ovide) peuvent se résumer comme ceci: «Baudelaire devient [...] son propre Ovide. Le poète latin n'est plus relégué dans le silence, comme dans «Alchimie de la douleur». Il n'est plus mis à distance par une critique de la raison pontique comme dans «Horreur sympathique». Il offre un modèle, «l'homme d'Ovide». Il le représente même, sinon par sa tendance à la plainte, du moins par son énergie de poète».

Afin de vérifier en quoi consiste, par rapport à Victor Hugo (notamment celui des Contemplations), la poétique du lyrisme baudelairien dans les années cruciales 1850-1860, PIERRE LAFORGUE («Les Contemplations», «Les Fleurs du Mal», ou le romantisme des 
années 1850, pp. 211-225) s'est spécialement concentré sur des poèmes tels que «L'Héautontimorouménos», "L'Irrémédiable», le troisième «Spleen» et «La Pipe». Comme il n'est pas question de résumer ici les nombreuses lignes de réflexion de cette étude, nous nous limitons à en souligner une parmi les plus essentielles, à savoir qu' avec Baudelaire entre en crise le lyrisme romantique des Lamartine, Hugo et Musset, cette crise étant due à la perte, chez le poète des Fleurs du Mal, d'«une certaine transitivité poétique, qui assignait au Je un objet en dehors de lui-même».

MARTINE LAVAUD (Gautier, Baudelaire et les 'bouquets séchés du romantisme', pp. 227-238) a étudié avec attention et équilibre «la circulation intertextuelle et poétique» qui lia Baudelaire au dédicataire des Fleurs du Mal, c'est-à-dire à l'auteur, entre autres, de Mademoiselle de Maupin et de La Comédie de la Mort. Ayant ramassé «les fleurs éparses du romantisme», Gautier et Baudelaire révèlent tous deux de frappantes affinités et, en même temps, d'indubitables dissonances, ce qui fait que l'un «tend à les travailler successivement, en des expériences esthétiques distinctes, pures et minérales, l'autre les jette toutes ensemble dans le creuset poétique, cherche à tisser dans leur discordance un réseau de correspondances, et réintroduisant la modernité, l'angoisse de la mort et de la chair, les nourrit d'une sève malade».

Partant de la conviction que le paratexte peut être considéré comme un signe d'une socialité du texte, JEAN-PIERRE BERTRAND \& PASCAL DURANT (Entre Gautier et Du Camp. D'une dédicace à l'autre: la position des Fleurs du Mal, pp. 239-255) ont voulu prouver le fondement de cette thèse en étudiant attentivement, aux deux extrémités des Fleurs $d u$ Mal, les deux dédicaces, respectivement à Théophile Gautier et à Maxime Du Camp, «dédicaces tutélaires» situées de façon symétrique au début et à la fin du recueil («Le Voyage»). L' «hypothèse conductrice» des auteurs consiste à démontrer que "c'est audelà de ces deux pôles de référence du moderne que se déploie la modernité des Fleurs et que se mesure leur force d'effraction et d'instauration». Pour ce faire, ils ont successivement reconsidéré les carrières de Gautier et de Du Camp pour en saisir d'abord la signification profonde et pour montrer ensuite combien la poésie baudelairienne se voulut moderne et se fit moderne aussi bien au-delà d'un «art pur tournant le dos à la 'Vie' «(Gautier) que d'une «poésie du contemporain» tournée vers l'avenir (Du Camp).

Cette dernière section se termine par l'intéressante contribution de LUC FRAISSE (Marcel Proust commente Les Fleurs du Mal, pp. 257-270). L'auteur s'est proposé - comme il l'affirme lui-même - de «lire Les Fleurs du Mal avec le regard de Marcel Proust» et d'«extraire de ses textes critiques un système d'interprétation de la poésie baudelairienne». De Contre Sainte-Beuve jusqu'à la chronique A propos de Baudelaire (publiée en juin 1921), il nous retrace en effet un riche parcours, tout au long duquel on peut connaître, dans leurs différents aspects, les réactions critiques de Proust lecteur des Fleurs. Le rapport entre la forme et le fond, entre la cruauté et la création, la technique du style, le classicisme de Baudelaire et, bien sûr, le temps, voilà les sujets principaux faisant l'objet de cette contribution, grâce à laquelle il nous est aisé de comprendre pourquoi l'auteur de la Recherche «voit inscrites dans Les Fleurs du Mal les traces de l'aphasie», ce qui nous «livrerait peut-être - conclut l'auteur - le secret et de la fin mortelle du poète et - dans un autre sens - de la fin dernière de la poésie».

L'intérêt majeur du présent volume nous semble résider surtout dans la grande variété des approches critiques et dans les perspectives nouvelles qui nous sont fréquemment 
ouvertes. Malgré certaines interprétations qui peuvent parfois paraître discutables, il s'agit là d' un ensemble de tout premier plan. 